Check for updates

Cite this: RSC Adv., 2018, 8, 23657

\title{
Multicolor carbon nanodots from food waste and their heavy metal ion detection application
}

\author{
Ying Zhou, ${ }^{a}$ Yao Liu, ${ }^{b}$ Yeqing Li, ${ }^{c}$ Ziying He, ${ }^{c}$ Quan Xu, (D) $* c$ Yusheng Chen, ${ }^{d}$ \\ Jason Street, ${ }^{e}$ Hao Guof and Michael Nelles ${ }^{* a}$
}

Multicolor carbon dots (C-dots) have excellent performance characteristics, high photoluminescence efficiency, ease of fabrication and low toxicity. C-dots have been used in a wide variety of fields including bioimaging, biomedicine, photocatalysis and environmental monitoring. The mass production of multicolor CDs using low-cost, facile methods is an important issue for future industrial applications. In this article, we reported a simple and highly effective way to prepare the multicolor C-dots and use them to detect heavy metal iron ions. Hydrochar acquired from food waste processed with hydrothermal carbonization (HTC) was used as the carbonaceous material for this process. Four colors of $\mathrm{C}$-dots were obtained and included blue, green, yellow and red. These multicolor $\mathrm{C}$-dots could be used as fluorescence probes with unique selectivity to detect the $\mathrm{Fe}^{3+}$ ion. The luminescence response ranged from 1 to $50 \mu \mathrm{M}$ with a correlation coefficient of 0.9968 . This discovery not only shows the high value-added products which can be obtained from food waste but can also lead to new developments in carbonaceous materials which can be used as "green resources".

Received 17th April 2018

Accepted 23rd June 2018

DOI: $10.1039 / \mathrm{c} 8 \mathrm{ra0} 3272 \mathrm{f}$

rsc.li/rsc-advances groups $^{7}$ have the potential to produce functional carbon-based materials which have applications as heavy metal adsorbents, soil additives, and electrode materials. ${ }^{8}$

In terms of carbonaceous nanomaterials, "green resources" have low toxicity when compared with semiconducting quantum dots and can be regarded as promising candidates for the production of photoluminescent materials. ${ }^{9}$ Among numerous kinds of nanomaterials such as carbon nanotubes, fullerenes and graphene, carbon dots (C-dots) have drawn much attention because of their photoelectrocatalytic efficiency, ease of fabrication and low cost. ${ }^{10} \mathrm{C}$-dots are quasi-spherical nanoparticles which are composed of a carbon skeleton with a diameter of less than $10 \mathrm{~nm} .{ }^{\mathbf{1 1}}$ The C-dots show excellent photoabsorption, photostability, photobleaching resistance, high water solubility, and chemical resistance properties with an ability to be easily functionalized. ${ }^{\mathbf{1 2}}$ Fluorescent nanomaterials derived from these C-dots made using FW can be used in bioimaging, biomedicine and environmental monitoring. ${ }^{13}$ The ability to improve fluorescence performance has been an area of great interest in last 10 years. ${ }^{14}$ In most studies concerning this topic, surface-modification has been regarded as the standard method for fluorescence improvements and includes functionalization, passivation and atom doping. There has not been a great deal of study concerning the fluorescent property modification with the use of carbonaceous materials from various sources, especially concerning food waste.

Hydrochar was produced from food waste using a mild hydrothermal temperature and was used as a carbon-rich precursor to synthesize C-dots in this study. A top-down method of synthesis was used to obtain multicolor C-dots. The effect that 
different hydrochar preparation techniques had on the fluorescence properties of C-dots was studied to determine the most advantageous methodology to use. C-dots have been used to detect mercury ions which are toxic for the environment and are human health hazards. In terms of the top-down method, the formation of C-dots occurs through exfoliating small particles from carbonaceous materials derived from methods including arc-discharge, laser ablation or electrochemical synthesis. ${ }^{15}$ C-dots obtained from this method may have weak luminescent properties, but are ideal concerning $\mathrm{sp}^{2}$ structures. ${ }^{\mathbf{1 6}}$ There are many influencing factors which affect the photoluminescence mechanism in C-dots and include molecular states, surface defects, size effect or the synergistic effect. ${ }^{17-19}$ Past studies show that photoluminescence emissions may be caused by surface or molecule states and an intrinsic band gap which is produced by confined $\mathrm{sp}^{2}$ conjugation in the core of C-dots. ${ }^{20}$ Carbon sources, synthetic methods and reaction conditions such as the type of solvent, temperature and $\mathrm{pH}$ are related to the multicolor emission of C-dots. Moreover, compared with quantum dots, surface groups make more of a contribution than the size effect concerning the photoluminescence color of C-dots. The unique photoluminescence properties of C-dots allow them to be used for applications involving ion detection including $\mathrm{Fe}^{3+}, \mathrm{Cu}^{2+}, \mathrm{Hg}^{2+}$ and $\mathrm{Cr}$ irons. ${ }^{21}$ Producing fluorescent probes with a high sensitivity and selectivity to detect toxic metal ions is important to help improve overall environmental conditions. ${ }^{22-24}$

\section{Results and discussion}

\section{Characteristics of precursor - hydrochar}

In this study, hydrochar was used as the precursor to synthesize multicolor C-dots. Fig. 1A shows the flow chart of the multicolor
C-dots production and the two-part process. The first part of the process involved hydrochar production which used food waste as carbon source and HTC technology as the treatment method. The second part of the process involved using the hydrochar as the precursor. The hydrochar was refluxed in concentrated acid and dialyzed with varying sizes to obtain C-dots with four colors: blue, green, yellow and red. Fig. 1B shows the carbon distribution involving different conditions of the HTC process. The distribution of the carbon in the solid phase (SP) did not increase as the temperature increased within the same retention time. The carbon distribution of SP-225 ${ }^{\circ} \mathrm{C}$ was less when compared to the SP-195 ${ }^{\circ} \mathrm{C}$ sample. All of the samples had a carbon distribution proportion above $60 \%$. Table 2 shows that the content of carbon in the hydrochar decreased as the temperature decreased. The hydrolysis of food waste is influenced by temperature and it would also effect processes including polymerization and aromatization, which modified the surface area or surface functional group of hydrochar differently.

\section{Characteristics of multicolor C-dots}

The morphology and structure of multicolor C-dots were characterized by transmission electron microscopy (TEM) and atomic force microscopy (AFM). In terms of AFM imaging, a dilute solution of multicolor C-dots were dropped on flat mica substrates. The TEM image indicated that green (Fig. 2B and F) and yellow (Fig. 2C and G) C-dots ranged in size from $2 \mathrm{~nm}$ to $12 \mathrm{~nm}$, respectively. The average diameters were $5.24 \mathrm{~nm}$ and $5.35 \mathrm{~nm}$, respectively. Both the green and yellow C-dots had a spherical shape and were separated in solution. The red (Fig. 2D and H) C-dots one had an average diameter of $6.26 \mathrm{~nm}$. The blue (Fig. 2A and E) C-dots had an average diameter of

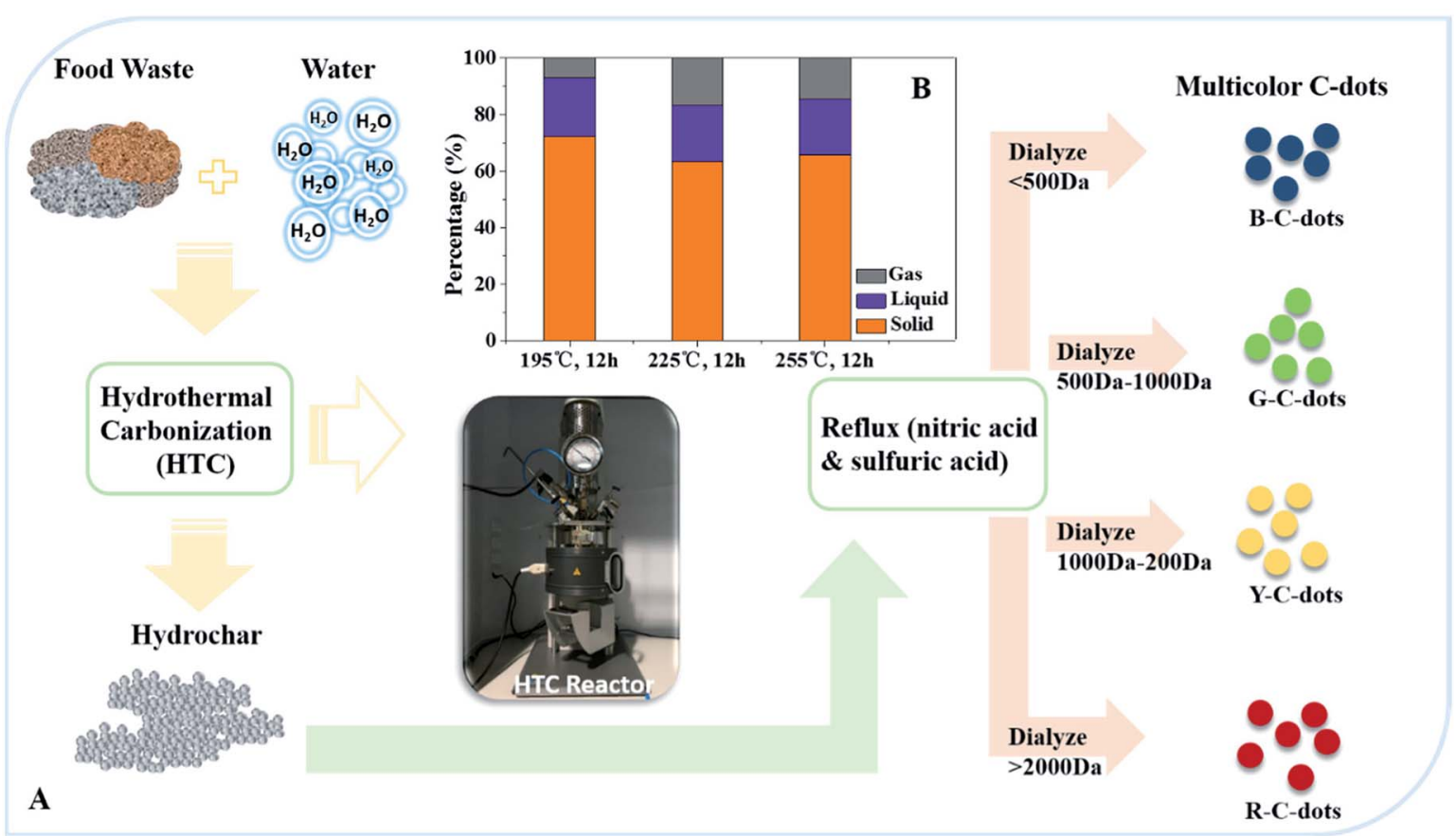

Fig. 1 (A) Flow chart of multicolor C-dots production. (B) Carbon distribution in gas, liquid and solid phase from the new precursor production process. 

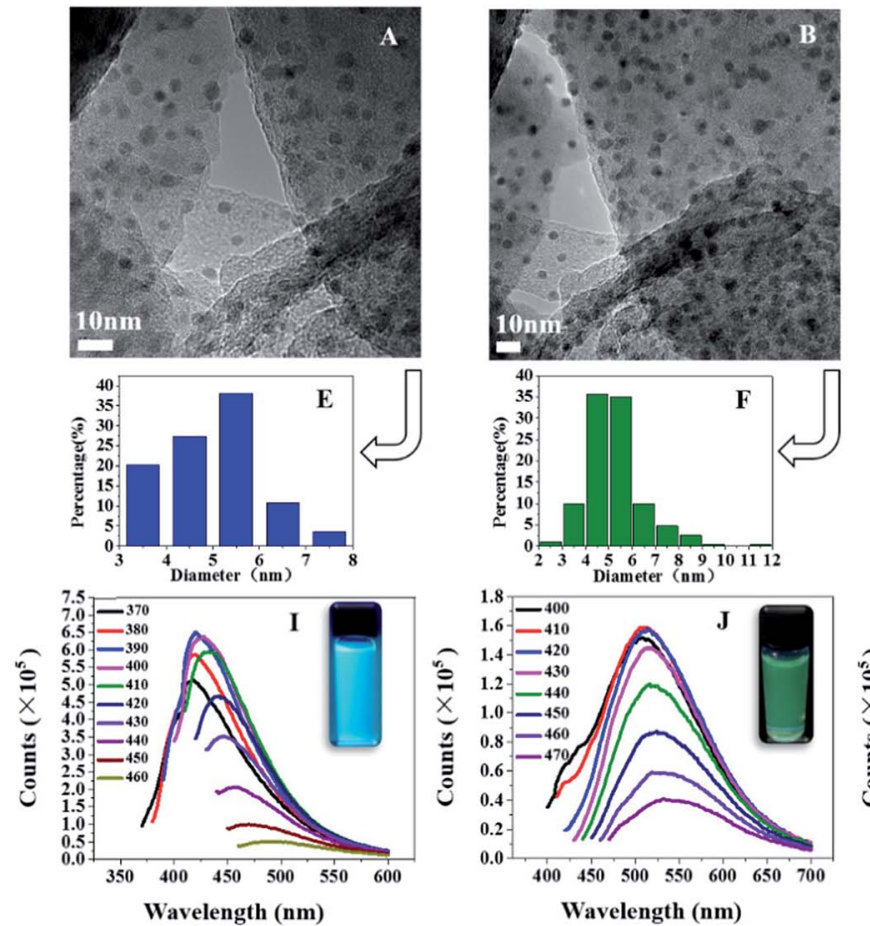
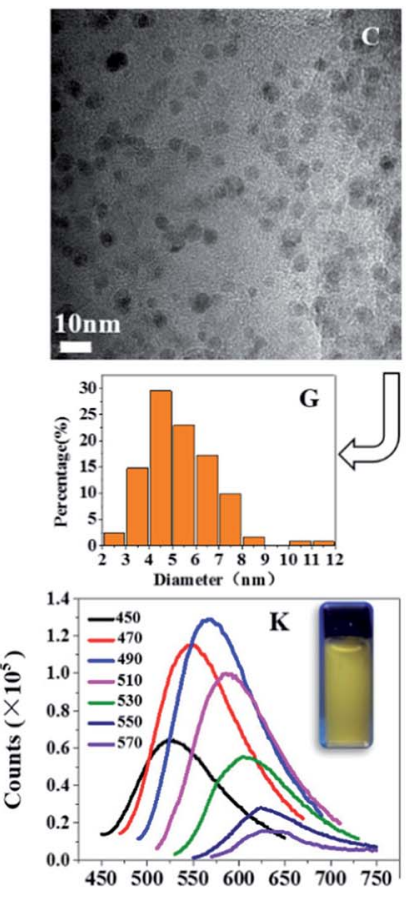

Wavelength (nm)
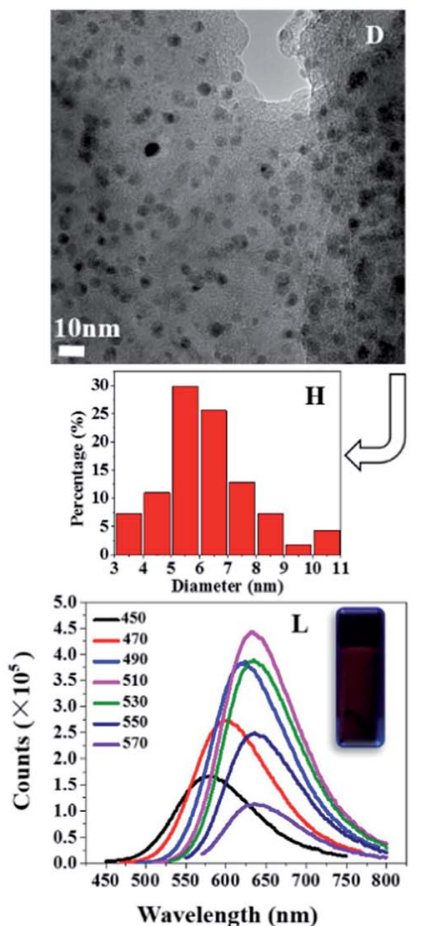

Fig. 2 TEM image of four color $C$-dots (A-D), size distribution of four color $C$-dots $(E-H)$, photoluminescence spectrum of four color $C$-dots $(I-L)$.

$5 \mathrm{~nm}$. The photoluminescence of C-dots were dialyzed into four groups: less than 500 Da (Fig. 2I), from 500 Da to $1000 \mathrm{Da}$ (Fig. 2J), from 1000-2000 Da (Fig. 2K), and more than $2000 \mathrm{Da}$ (Fig. 2L). The samples were irradiated at varying wavelengths and studied in detail. Fig. 2J shows that as the $\lambda_{\mathrm{ex}}$ varied from 400-470 $\mathrm{nm}$, the peak position was not altered and only the intensity of $\lambda_{\mathrm{ex}}$ decreased rapidly. The fluorescence color of these C-dots was green, and the result obviously showed the excitement independent property of the C-dots. This was primarily due to the homogeneous surface structure. Fig. 2I shows a slight shift of the peak position as the $\lambda_{\text {ex }}$ varied from $370-460 \mathrm{~nm}$, and the fluorescence color for this sample was blue. The fluorescence peak positions of C-dots with a yellow and red color varied with the various intensities of the irradiated $\lambda_{\text {ex }}$ (Fig. $2 \mathrm{~K}$ and $\mathrm{L}$ ). The photoluminescence quantum yield was $28 \%, 18 \%, 10 \%, 6 \%$, representing blue C-dots, green Cdots, yellow C-dots, red C-dots, respectively. In the lowerenergy region, the yellow C-dots spectra exhibit distinct absorption bands at 420 and the green one also has absorption around $440 \mathrm{~nm}$, indicating that these samples possessed different surface states ${ }^{25,26}$ (Fig. 3A).

The FTIR spectrum specified the different functional groups found in the C-dots (Fig. 3C-F). With the exception of the green C-dots, the others had peaks found at $1250 \mathrm{~cm}^{-1}$ (which belonged to the $\mathrm{C}-\mathrm{O}$ group) and $1629 \mathrm{~cm}^{-1}$ (which belonged to the $\mathrm{C}=\mathrm{O}$ group). With the exception of the blue C-dots, the others had peaks located at $3424 \mathrm{~cm}^{-1}$ (which belonged to the $\mathrm{O}-\mathrm{H}$ group) and $1500 \mathrm{~cm}^{-1}$ (which refers to a phenolic group). The $\mathrm{C}-\mathrm{N}$ band at $1012 \mathrm{~cm}^{-1}$ and the $\mathrm{C}=\mathrm{N}$ band at $1549 \mathrm{~cm}^{-1}$ appear in the spectrum for the C-dots.
To further gain insight into how the functional groups or chromophore affects the absorption and photoluminescence of C-dots, the C1s (Fig. 4A), N1s (Fig. 4B) and O1s (Fig. 4C) XPS spectra for four color C-dots were carefully examined. In the O1s (Fig. 4A) XPS spectrum, the four color C-dots have obvious change in the peaks of 287.8 , the blue C-dots has a distinct peak at $287.8 \mathrm{eV}(\mathrm{C}=\mathrm{O})$, while there is no peak found in red C-dots, indicating that the oxidation state of carbon was related to the absorption and photoluminescence of C-dots. The XPS spectrum of C1s is consistent with FTIR analysis, providing further evidence for oxidation state $\mathrm{C}$ was introduced in the reaction, serving as the passivation spots for the high fluorescence. In the N1s (Fig. 4B) XPS spectrum the peaks of the four color C-dots are shifted from 398.4 to 397.8 indicating the change in the chemical state of nitrogen element from pyrrolic $\mathrm{N}$ to pyridinic $\mathrm{N}$. The nitrogen also played a critic role in the absorption. The O1s XPS spectrum (Fig. 4C) of the four color Cdots were consistent with C1s analysis.

Those C-dots have high stability and a simple synthetic route for production and have found broad applications in various fields including sensor development. These materials could be used for the unique selectivity of iron ions according to their luminescence response. Here we use the green C-dots were selective for quenching ions of $\mathrm{Cu}^{2+}, \mathrm{Fe}^{3+}$, and $\mathrm{Hg}^{2+}$ (no EDTA, F0/F1, Fig. 5A). However, the ability of the C-dots to quench the ions is difficult to recognize when the ions coexist in the solution. When the solution contained ethylenediaminetetraacetic acid (EDTA, F2/F3, Fig. 5B) the C-dots were found to be dramatically quenched only after the addition of $\mathrm{Fe}^{3+}$. The other ions mentioned above did not have the same response to the 

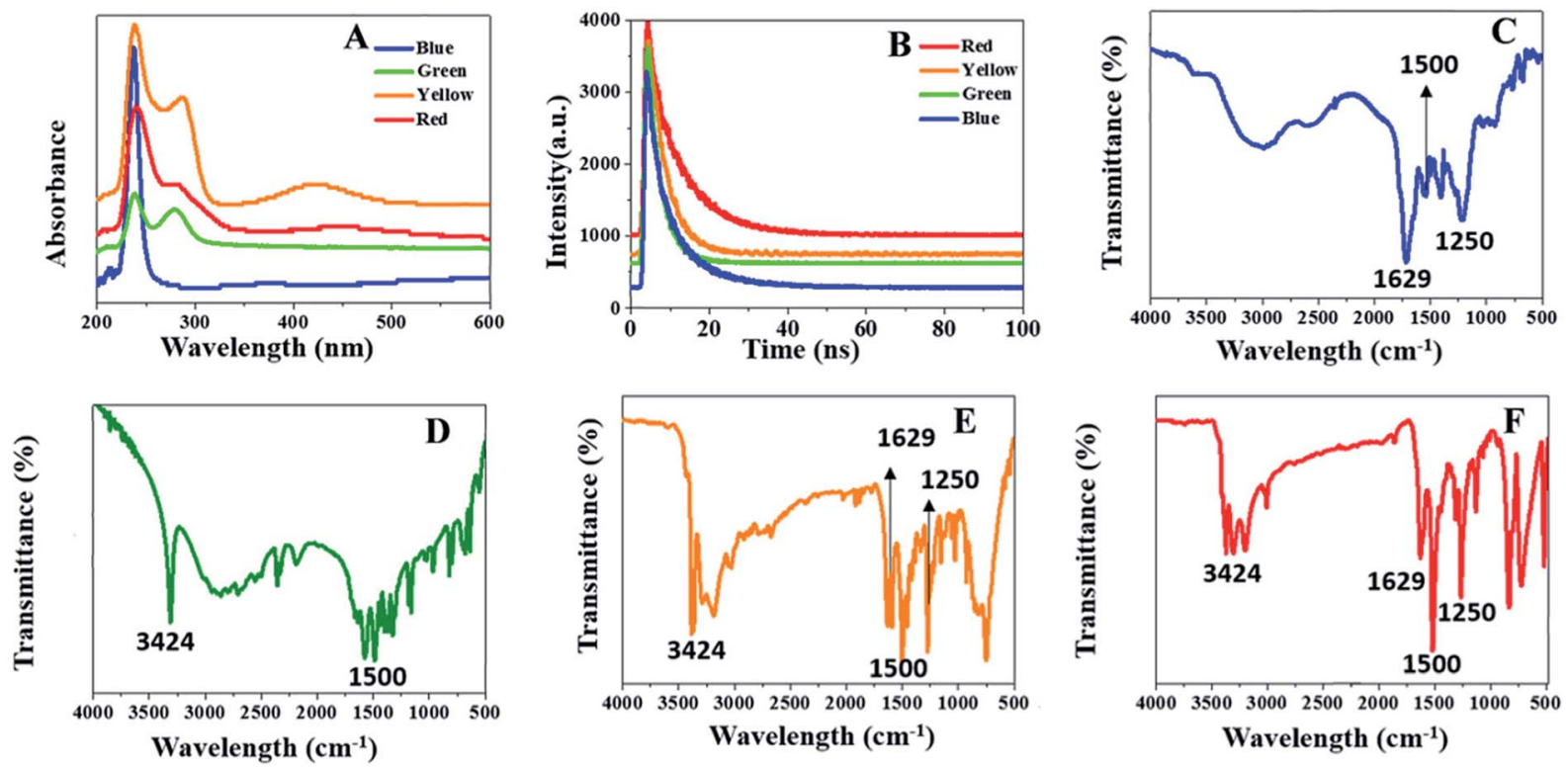

Fig. 3 Optical absorption spectrum of C-dots (A). Life curve for of C-dots (B), FTIR of four colors C-dots (blue, green, yellow and red) (C-F).

quenching process. The phenomenon can be ascribed to the interactions between the surface groups of C-dots and the metal ions having fewer interactions between EDTA and the metal ions $\left(\mathrm{Cu}^{2+}, \mathrm{Hg}^{2+}\right.$ and $\left.\mathrm{Ce}^{3+}\right)$. Further investigation was carried out (F4 based on F3, Fig. 5C) and results indicated that other ions had negligible influence on the fluorescence. Thus, these results showed that the C-dots have high accuracy and uniqueselectivity to the iron ion in the EDTA solution. The possible reason that quenching of fluorescence by $\mathrm{Fe}^{3+}$ ions was that due to the oxygenous groups on the surface which form an unique structure at the molecular level. Similar to chelation interaction, this unique structure and $\mathrm{Fe}^{3+}$ ions can serve as receptors and ligand, respectively. The steric hindrance impact of unique structure lead to the high selectivity for $\mathrm{Fe}^{3+}$ ions versus other ions, which also impact the fluorescence of C-dots. The coordinated $\mathrm{Fe}-\mathrm{CDs}$ complex was favorable for the charge transfer, and also inhibited the excitons recombination, thus leading to the corresponding PL quenching. ${ }^{27,28}$ The fluorescence intensity of the C-dots solution decreased as the concentration of $\mathrm{Fe}^{3+}$ increased. The resulting calibration curve for $\mathrm{Fe}^{3+}$ displayed good linearity for concentrations ranging from 1 to $50 \mu \mathrm{M}$ with a correlation coefficient of 0.9968 (Fig. 5D).

\section{Hydrothermal carbonization}

Food waste with a mass of $250 \mathrm{~g}$ and water with a mass of $450 \mathrm{~g}$ were added into a $1 \mathrm{~L}$ autoclave. A fixed temperature was used $\left(195{ }^{\circ} \mathrm{C}, 225{ }^{\circ} \mathrm{C}\right.$ and $\left.255^{\circ} \mathrm{C}\right)$ for $12 \mathrm{~h}$, and the hydrothermal products were separated using vacuum filtration. The solid phase products were placed into an oven at $105{ }^{\circ} \mathrm{C}$ for $24 \mathrm{~h}$. The hydrochar was sealed and used as the precursor for the following multicolor C-dots production. The properties of the food waste are shown in Table 1.

\section{Synthesis of multicolor C-dots}

Multicolor C-dots were synthesized using a top-down method. At room temperature, $2 \mathrm{~g}$ hydrochar with $20 \mathrm{ml}$ concentrated nitric acid and $60 \mathrm{ml}$ concentrated sulfuric acid were added into a $100 \mathrm{ml}$ flask in sequence. The flask was inserted into a $700 \mathrm{~W}$ ultrasonic cleaning system for $2 \mathrm{~h}$ and the dispersed liquid products were collected. The liquid products were refluxed in an oil bath at $100{ }^{\circ} \mathrm{C}$ for $10 \mathrm{~h}$ and then cooled to room temperature. The product was neutralized by adding $\mathrm{NaOH}$ and distilled water. The products were later dialyzed for $72 \mathrm{~h}$ using three distinct sizes of dialysis
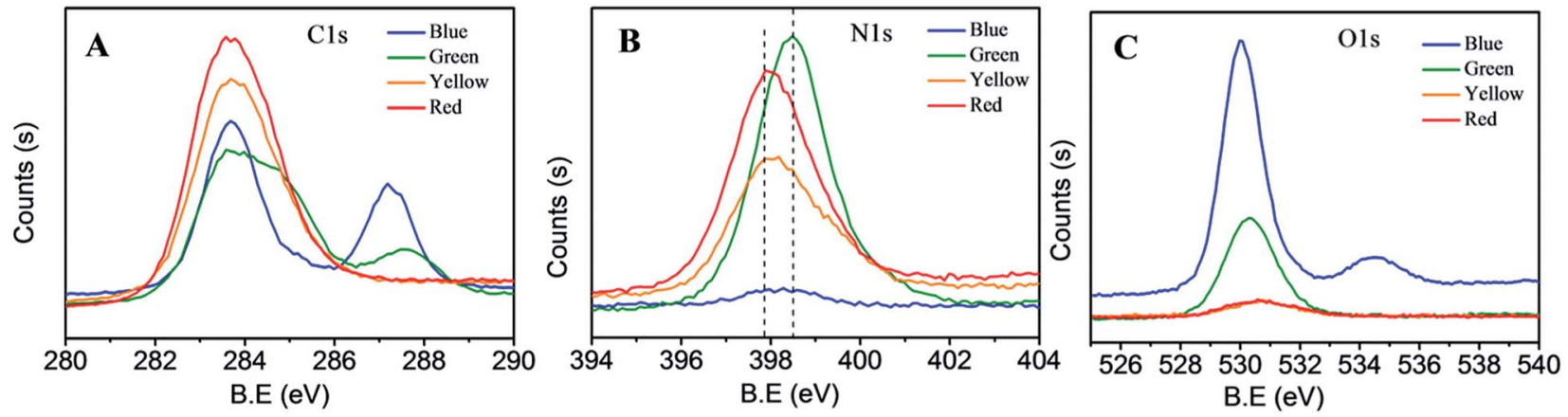

Fig. 4 (A-C) High-resolution C1s, N1s and O1s XPS spectra for four color C-dots. 

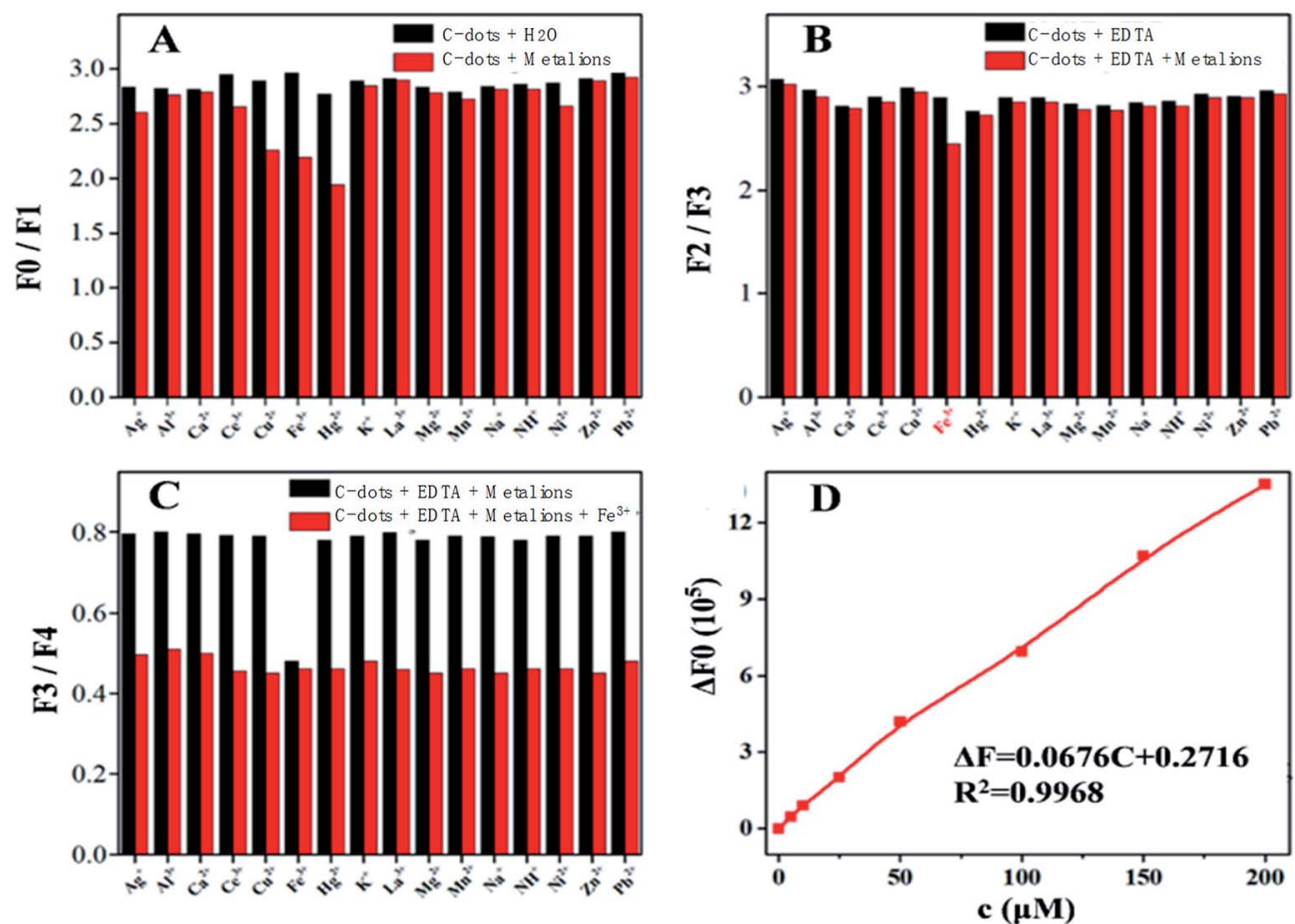

Fig. 5 The change of fluorescence intensity at $530 \mathrm{~nm}$ for C-dots in the presence of deionized water F0 and various metal ions F1 (A), EDTA solution F2 and F2 addition of various metal ions denoted as F3 (B), F3 and F3 addition of the iron ion denoted as F4 (C), the change of fluorescence intensity of $\mathrm{C}$-dots solution versus the concentration of $\mathrm{Fe}^{3+}(\mathrm{D})$. The change of fluorescence intensity of $\mathrm{C}$-dots solution versus the concentration of $\mathrm{Fe}^{3+}$.

Table 1 Elemental analysis of hydrochar

\begin{tabular}{lccccc}
\hline Condition & $\mathrm{C}(\%)$ & $\mathrm{H}(\%)$ & $\mathrm{N}(\%)$ & $\mathrm{O}^{*}(\%)$ & Ash (\%) \\
\hline Hydrochar-195 ${ }^{\circ} \mathrm{C}, 12 \mathrm{~h}$ & 69.76 & 7.03 & 3.64 & 17.73 & 1.34 \\
Hydrochar-225 ${ }^{\circ} \mathrm{C}, 12 \mathrm{~h}$ & 72.88 & 7.06 & 3.85 & 14.41 & 1.30 \\
Hydrochar-255 ${ }^{\circ} \mathrm{C}, 12 \mathrm{~h}$ & 74.75 & 7.22 & 3.87 & 12.36 & 1.30
\end{tabular}

tube orifice sizes including $500 \mathrm{Da}, 1000 \mathrm{Da}$ and $2000 \mathrm{Da}$ to produce blue, green, yellow and red C-dots.

\section{Analytical methods}

The content of cellulose, hemicellulose, and lignin were determined with an ANKOM A200 Filter Bag Technique (FBT). The elemental content of hydrochar were analyzed by a Vario EL III elemental analyzer. The oxygen content was found by subtracting the sum of the other elements and ash content from
$100 \%$. The characterized morphology of multicolor C-dots was found using Transmission Electron Microscopy (TEM) with equipment from the Japan Electron Optics Laboratory Ltd. (Model JEM-2100F). Atomic Force Microscope (AFM) (Bruker Scan-Dimension-Icon system) was adopted to further characterize the size and morphology of the samples. The optical performance of the multicolor C-dots was based on the photoluminescence spectrum, optical absorption spectrum, and life curve. These were investigated using a multipurpose fluorescence spectrophotometer (FS5) from Techcomp (China) Ltd. The sample from the original C-dots solution was diluted at a ratio of $10: 1$ and inserted in a quartz fluorescence cuvette. The emission characteristics were investigated at diverse excitation irradiation. The samples were investigated with using Fourier transform infrared (FTIR) spectroscopy. A Bruker Vertex $70 \mathrm{v}$ was used to analyze the different functional groups in the multicolor C-dots.

Table 2 Properties of food waste

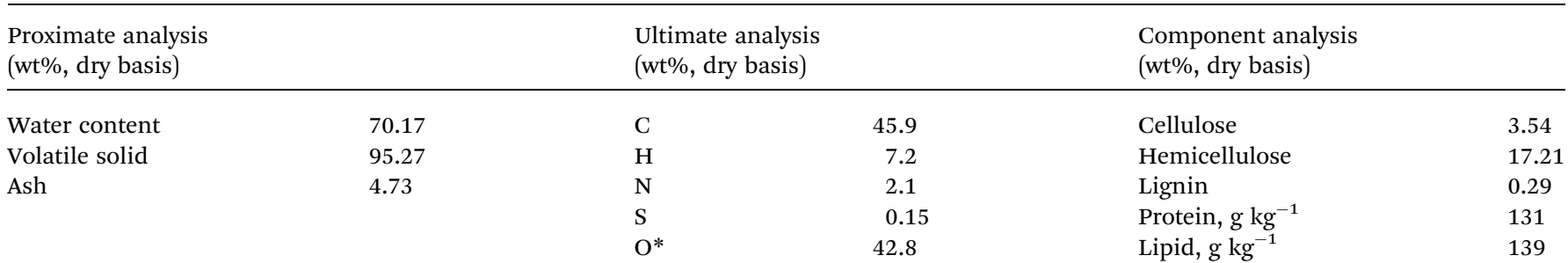




\section{Conclusions}

In conclusion, we have developed a facile and low-cost approach to prepare multicolor C-dots-blue, green, yellow and red with varying nanometer diameters using food waste as a precursor. The most vital step of preparation involved the treatment using HTC technology, an eco-friendly method to dispose of the food waste. The products of C-dots were used as fluorescence probes to detect heavy metal ions. The samples could be used for the unique selectivity of the $\mathrm{Fe}^{3+}$ ion according to the luminescence response ranging from 1 to $50 \mu \mathrm{M}$ with a correlation coefficient of 0.9968. The multicolor C-dots with a characteristic optical performance are excellent candidates for environmental, sensor, and biological applications. This discovery could lead to improving the value of food waste while encouraging new developments in economically friendly, carbon materials.

\section{Conflicts of interest}

There are no conflicts to declare.

\section{Acknowledgements}

We thank Beijing Nova Program Interdisciplinary Studies Cooperative project (No. Z181100006218138), Science Foundation of China University of Petroleum-Beijing (No. 2462018BJC004), State Key Laboratory of Petroleum \& Petrochemical Pollution Control and Treatment (No. PPC2017015) for the support. This material is supported by the National Institute of Food and Agriculture, U.S. Department of Agriculture, and McIntire Stennis under accession number 1009735.

\section{Notes and references}

1 X. Jia, B. Xi, M. Li, T. Xia, Y. Hao, D. Liu and J. Hou, J. Cleaner Prod., 2017, 152, 364-368.

2 M. Mourad, J. Cleaner Prod., 2016, 126, 461-477.

3 Y. Gao, B. Yu, X. Wang, Q. Yuan, H. Yang, H. Chen and S. Zhang, Energy, 2015, 88, 139-148.

4 Y. Gao, B. Yu, K. Wu, Q. Yuan, X. Wang and H. Chen, BioResources, 2016, 11, 4113-4133.

5 X. Chen, Q. Lin, R. He, X. Zhao and G. Li, Bioresour. Technol., 2017, 241, 236-243.

6 N. Zhou, H. Chen, J. Xi, D. Yao, Z. Zhou, Y. Tian and X. Lu, Bioresour. Technol., 2017, 232, 204-210.

7 E. Unur, S. Brutti, S. Panero and B. Scrosati, Microporous Mesoporous Mater., 2013, 174, 25-33.

8 K. Wu, Y. Gao, G. Zhu, J. Zhu, Q. Yuan, Y. Chen, M. Cai and L. Feng, J. Anal. Appl. Pyrolysis, 2017, 127, 335-342.
9 J. Jeong, J. Jung, M. Choi, J. W. Kim, S. J. Chung, S. Lim, H. Lee and B. H. Chung, Adv. Mater., 2012, 24, 1999-2003.

10 Q. Xu, P. Pu, J. Zhao, C. Dong, C. Gao, Y. Chen, J. Chen, Y. Liu and H. Zhou, J. Mater. Chem. A, 2015, 3, 542-546.

11 Q. Xu, Y. Liu, C. Gao, J. Wei, H. Zhou, Y. Chen, C. Dong, T. S. Sreeprasad, N. Li and Z. Xia, J. Mater. Chem. C, 2015, 3, 9885-9893.

12 S. Y. Park, H. U. Lee, E. S. Park, S. C. Lee, J. W. Lee, S. W. Jeong, C. H. Kim, Y. C. Lee, Y. S. Huh and J. Lee, ACS Appl. Mater. Interfaces, 2014, 6, 3365-3370.

13 Q. Xu, Y. Liu, R. Su, L. Cai, B. Li, Y. Zhang, L. Zhang, Y. Wang, Y. Wang, N. Li, X. Gong, Z. Gu, Y. Chen, Y. Tan, C. Dong and T. S. Sreeprasad, Nanoscale, 2016, 8, 1791917927.

14 P. K. Sarswat and M. L. Free, Phys. Chem. Chem. Phys., 2015, 17, 27642-27652.

15 L. Li, G. Wu, G. Yang, J. Peng, J. Zhao and J.-J. Zhu, Nanoscale, 2013, 5, 4015.

16 Q. Xu, T. Kuang, Y. Liu, L. Cai, X. Peng, T. Sreenivasan Sreeprasad, P. Zhao, Z. Yu and N. Li, J. Mater. Chem. B, 2016, 4, 7204-7219.

17 V. Gude, A. Das, T. Chatterjee and P. K. Mandal, Phys. Chem. Chem. Phys., 2016, 18, 28274-28280.

18 S. Hu, Chem. Rec., 2016, 16, 219-230.

19 B. Li, Y. Guo, A. Iqbal, Y. Dong, W. Li, W. Liu, W. Qin and Y. Wang, RSC Adv., 2016, 6, 107263-107269.

20 M. Shamsipur, A. Barati and S. Karami, Carbon, 2017, 124, 429-472.

21 S. Y. Lim, W. Shen and Z. Gao, Chem. Soc. Rev., 2015, 44, 362381.

22 Y. Liu, C. Liu and Z. Zhang, Appl. Surf. Sci., 2012, 263, 481485.

23 Q. Xu, L. Ding, Y. Wen, W. Yang, H. Zhou, N. Li, X. Chen, J. Street, Y. Chen, A. Zhou and W. Ong, J. Mater. Chem. C, 2018, 6, 6360-6369.

24 Q. Xu., R. Su, Y. Chen, S. Sreenivasan, N. Li, X. Zheng, J. Zhu, H. Pan, W. Li, C. Xu., Z. Xia and L. Dai, ACS Appl. Nano Mater., 2018, 1, 1886-1893.

25 H. Nie, M. Li, Q. Li, S. Liang, Y. Tan, L. Sheng, W. Shi and S. X.-A. Zhang, Chem. Mater., 2014, 26, 3104-3112.

26 H. Ding, S.-B. Yu, J.-S. Wei and H.-M. Xiong, ACS Nano, 2016, 10, 484-491.

27 L. Xu, W. Mao, J. Huang, S. Li, K. Huang, M. Li, J. Xia and Q. Chen, Sens. Actuators, B, 2016, 230, 54-60.

28 H. Xu, S. Zhou, L. Xiao, H. Wang, S. Li and Q. Yuan, J. Mater. Chem. C, 2015, 3, 291-297. 